\title{
Activation of the $\beta$-Adrenoceptor-Protein Kinase A Signaling Pathway within the Ventral Bed Nucleus of the Stria Terminalis Mediates the Negative Affective Component of Pain in Rats
}

\author{
Satoshi Deyama, ${ }^{1,2}$ Takahiro Katayama, ${ }^{1}$ Atsushi Ohno, ${ }^{1}$ Takayuki Nakagawa, ${ }^{2}$ Shuji Kaneko, ${ }^{2}$ Taku Yamaguchi, ${ }^{3}$ \\ Mitsuhiro Yoshioka, ${ }^{3}$ and Masabumi Minami ${ }^{1}$ \\ ${ }^{1}$ Department of Pharmacology, Graduate School of Pharmaceutical Sciences, Hokkaido University, Sapporo 060-0812, Japan, ${ }^{2}$ Department of Molecular \\ Pharmacology, Graduate School of Pharmaceutical Sciences, Kyoto University, Kyoto 606-8501, Japan, and ${ }^{3}$ Deparment of Neuropharmacology, Graduate \\ School of Medicine, Hokkaido University, Sapporo 060-8638, Japan
}

\begin{abstract}
Pain is an unpleasant sensory and emotional experience. The neural systems underlying the sensory component of pain have been studied extensively, but we are only beginning to understand those underlying its affective component. The bed nucleus of the stria terminalis (BNST) has been implicated in stress responses and negative affective states, such as anxiety, fear, and aversion. Recently, we demonstrated the crucial role of the BNST in the negative affective component of pain using the conditioned place aversion (CPA) test. In the present study, we investigated the involvement of the $\beta$-adrenoceptor-protein kinase A (PKA) signaling pathway within the BNST, in particular, within the ventral part of the BNST (vBNST), in pain-induced aversion in male Sprague Dawley rats. In vivo microdialysis showed that extracellular noradrenaline levels within the vBNST were significantly increased by intraplantar formalin injection. Using the CPA test, we found that intra-vBNST injection of timolol, a $\beta$-adrenoceptor antagonist, dose-dependently attenuated the intraplantar-formalin-induced CPA (F-CPA) without reducing nociceptive behaviors. Experiments with subtype-selective antagonists demonstrated the essential role of $\beta_{2}$-adrenoceptors in F-CPA. Intra-vBNST injection of isoproterenol, a $\beta$-adrenoceptor agonist, dosedependently produced CPA even in the absence of noxious stimulation. This isoproterenol-induced CPA was reversed by the coinjection of Rp-cyclic adenosine monophosphorothioate (Rp-cAMPS), a selective PKA inhibitor. Furthermore, intra-vBNST injection of RpcAMPS dose-dependently attenuated the F-CPA. Together, these results suggest that PKA activation within the vBNST via the enhancement of $\beta$-adrenergic transmission is important for the negative affective component of pain.
\end{abstract}

Key words: bed nucleus of the stria terminalis; conditioned place aversion; emotion; noradrenaline; protein kinase A; pain

\section{Introduction}

Pain is a complex experience composed of sensory and affective components. Although the neural systems responsible for the sensory component of pain have been studied extensively, the neural basis of the affective component is not well understood. Recently, behavioral studies examined the neural circuits and mechanisms underlying the negative affective component of pain. Using a conditioned place paradigm, Johansen et al. (2001) demonstrated that excitotoxic lesions of the anterior cingulate cortex suppressed conditioned place aversion (CPA) induced by the intraplantar injection of formalin, without reducing

Received Dec. 13, 2007; revised May 12, 2008; accepted June 18, 2008.

This work was supported by a Grant-in-Aid for Scientific Research (B) 19390149 from the Japan Society for the Promotion of Science (M.M.) and grants from the Naito Foundation (M.M.), Suzuken Memorial Foundation (M.M.), and Takeda Science Foundation (M.M.).

Correspondence should be addressed to Dr. Masabumi Minami, Department of Pharmacology, Graduate School of Pharmaceutical Sciences, Hokkaido University, Sapporo 060-0812, Japan. E-mail: mminami@pharm. hokudai.ac.jp.

DOI:10.1523/JNEUROSCI.1480-08.2008

Copyright $\odot 2008$ Society for Neuroscience $\quad$ 0270-6474/08/287728-09\$15.00/0 formalin-induced nociceptive behaviors. A similar experimental protocol showed that the central or basolateral amygdaloid nuclei are differently involved in intraplantar-formalin-induced CPA (F-CPA) and intraperitoneal acetic acid-induced CPA (A-CPA) (Tanimoto et al., 2003). The findings suggest the important roles of these brain regions in the affective component of pain. Moreover, glutamatergic transmission, especially via NMDA receptors, within these regions has been reported to be crucial to paininduced aversion (Johansen and Fields, 2004; Lei et al., 2004; Deyama et al., 2007b). In addition to these brain areas, Deyama et al. (2007a) recently reported the involvement of the bed nucleus of the stria terminalis (BNST) in the affective component of pain. Bilateral excitotoxic lesions of the BNST significantly attenuated F-CPA and A-CPA without reducing nociceptive behaviors. However, the neurochemicals involved in the signal transmission for pain-induced aversive responses within this brain region have not been identified.

The BNST, especially its ventral part (vBNST), is densely innervated by noradrenergic fibers arising mainly from the nuclei of the solitary tract (including the A2 cell group) and caudal 
ventrolateral medulla (including the A1 cell group) (Woulfe et al., 1990; Forray et al., 2000). Noradrenergic transmission within the vBNST is important for mediating negative emotions such as fear and anxiety. Specifically, blockade of $\beta$-adrenoceptors in the vBNST attenuates acute restraint stress-induced, anxiety-like behaviors (Cecchi et al., 2002) and morphine withdrawal-induced CPA (Delfs et al., 2000). These findings suggest that enhanced $\beta$-adrenergic transmission within the vBNST plays an important role in negative affective states. Thus, we first examined the involvement of intra-vBNST noradrenergic transmission via $\beta$-adrenoceptors in pain-induced aversion using a conditioned place paradigm in rats. $\beta$-Adrenoceptors couple to adenylate cyclases to activate a protein kinase A (PKA), but no direct evidence exists for the involvement of the $\beta$-adrenoceptor-PKA signaling pathway in the affective component of pain. Thus, we examined the effect of intra-vBNST administration of a selective PKA inhibitor on pain-induced aversion and found that activation of PKA by enhanced $\beta$-adrenergic transmission within the vBNST plays a crucial role in pain-induced aversion.

\section{Materials and Methods}

Animals. Male Sprague Dawley rats (180-240 g; Japan SLC) were used. Rats were maintained in a constant ambient temperature $\left(22 \pm 1^{\circ} \mathrm{C}\right)$ under a $12 \mathrm{~h}$ light/dark cycle with food and water available ad libitum. All experiments were performed with the approval of the Institutional Animal Care and Use Committee at Hokkaido University and Kyoto University.

Drugs. Timolol, a $\beta$-adrenoceptor antagonist, betaxolol, a $\beta_{1}$ adrenoceptor antagonist, (+)-1-[2,3-(dihydro-7-methyl-1 $H$-inden-4yl)oxy]-3-[(1-methylethyl)amino]-2-butanol (ICI118,551), a $\beta_{2}$ adrenoceptor antagonist, (-)-isoproterenol, a $\beta$-adrenoceptor agonist, and Rp-cyclic adenosine monophosphorothioate (Rp-cAMPS), a selective PKA inhibitor, were purchased from Sigma. These drugs were dissolved in sterile PBS or sterile saline.

Immunostaining for dopamine- $\beta$-hydroxylase. Rats were deeply anesthetized with sodium pentobarbital and perfused transcardially with PBS followed by $4 \%$ paraformaldehyde in $0.1 \mathrm{M}$ phosphate buffer. Brains were removed, postfixed in the same fixative overnight, cryoprotected with $20 \%$ sucrose in $0.1 \mathrm{M}$ phosphate buffer for $48 \mathrm{~h}$ at $4^{\circ} \mathrm{C}$, and then frozen in powdered dry ice. Coronal sections ( $30 \mu \mathrm{m}$ thick) were cut in a cryostat and collected in PBS to be processed immunohistochemically as freefloating sections. The atlas of Paxinos and Watson (1998) was used to identify the brain region. Sections were blocked in $4 \%$ normal goat serum in PBS containing $0.3 \%$ Triton X-100 (PBST) for $1 \mathrm{~h}$ at room temperature, and incubated with primary monoclonal mouse anti-dopamine- $\beta$ hydroxylase (DBH) antibody (1:1000; Millipore) in PBST containing 4\% normal goat serum overnight at room temperature. After three washes in PBST, the sections were incubated with Alexa Fluor 488-conjugated goat anti-mouse IgG (1:200; Invitrogen) in PBST containing 4\% normal goat serum for $1 \mathrm{~h}$ at room temperature. After three washes in PBST, the sections were mounted on slides and coverslipped using Vectashield (Vector Laboratories). Immunofluorescence was visualized and photographed using a CCD camera (Keyence) mounted on a fluorescence microscope (Olympus).

In vivo microdialysis. Under sodium pentobarbital anesthesia $(50 \mathrm{mg} /$ $\mathrm{kg}$, i.p.), each rat was unilaterally implanted with a microdialysis guide cannula [outer diameter (o.d.), $0.5 \mathrm{~mm}$; AG-7; Eicom] $1.0 \mathrm{~mm}$ above the vBNST $(-0.3 \mathrm{~mm}$ rostral, $1.6 \mathrm{~mm}$ lateral, and $6.7 \mathrm{~mm}$ ventral to the bregma) (Paxinos and Watson, 1998). After surgery, the rats were individually housed in cages during the recovery period for at least $1 \mathrm{~d}$. Microdialysis experiments were performed in unanesthetized and freely moving rats. Ringer-primed microdialysis probes (dialysis membrane: length, $1.0 \mathrm{~mm}$; o.d., $0.22 \mathrm{~mm}$; A-I-7-01; Eicom) were inserted through the guide cannula and continuously perfused with normal Ringer's solution [containing (in $\mathrm{mm}$ ) $147 \mathrm{Na}^{+}, 4 \mathrm{~K}^{+}, 2.3 \mathrm{Ca}^{2+}, 155.6 \mathrm{Cl}^{-}$] at a constant flow rate of $1 \mu \mathrm{l} / \mathrm{min}$. The tip of the microdialysis probe protruded $1.0 \mathrm{~mm}$ from the tip of the guide cannula to reach the vBNST. The rats were then placed in a Plexiglas chamber (width $\times$ length $\times$ height: $30 \times 30 \times 35 \mathrm{~cm})$. After a stabilization period $(>2 \mathrm{~h}), 1115 \mathrm{~min}$ dialysates were collected. The first three samples were baseline samples. Immediately after collection of the last baseline sample, each rat was given an intraplantar injection of $2 \%$ formalin or saline (100 $\mu \mathrm{l})$ into the right hind paw. In some experiments, neuronal cells were directly stimulated by perfusing a high- $\mathrm{K}^{+}$solution (concentration of $\mathrm{K}^{+}, 50 \mathrm{~mm}$ ) in the presence or absence of $\mathrm{Ca}^{2+}$.

Each dialysate sample was separated in a liquid chromatography column [Eicompak CAX; $2.0 \mathrm{~mm}$ inner diameter (i.d.) $\times 200 \mathrm{~mm}$; Eicom] at $35^{\circ} \mathrm{C}$ with $0.1 \mathrm{M}$ ammonium acetate buffer, $\mathrm{pH} 6.0$, containing $0.05 \mathrm{M}$ sodium sulfate, $50 \mathrm{mg} / \mathrm{L}$ ethylene diamine tetraacetic acid, and $30 \%$ methanol at a constant flow rate of $0.25 \mathrm{ml} / \mathrm{min}$. Noradrenaline contents in the dialysates were measured with an electrochemical detector (HTEC-500; Eicom) with a working electrode set at $+450 \mathrm{mV}$ versus an $\mathrm{Ag} / \mathrm{AgCl}$ reference electrode. Chromatogram peaks were analyzed using the PowerChrom data recording system. Under this condition, three major neuronal monoamines, namely noradrenaline, dopamine, and serotonin were separated (supplemental Fig. $1 \mathrm{~A}$, available at www. jneurosci.org as supplemental material). The retention times of these monoamines were 5.3, 6.8, and $12.3 \mathrm{~min}$, respectively. The retention time of noradrenaline in dialysate samples matched that of an authentic standard (supplemental Fig. $1 B, C$, available at www.jneurosci.org as supplemental material).

Data are expressed as the means \pm SEM of the percentage basal value, which was calculated as an average of three consecutive dialysates before intraplantar injection or high $\mathrm{K}^{+}$stimulation. Area under the curve (AUC) values for noradrenaline levels during the $0-120 \mathrm{~min}$ period were calculated, and data are expressed as the means \pm SEM of the percentage of the AUC value in the saline-injected control group.

Microinjection. Under sodium pentobarbital anesthesia $(50 \mathrm{mg} / \mathrm{kg}$, i.p.), rats were implanted bilaterally with 25 -gauge stainless-steel guide cannulas (o.d., $0.5 \mathrm{~mm}$; i.d., $0.22 \mathrm{~mm}) 1.5 \mathrm{~mm}$ above the vBNST $(-0.3$ $\mathrm{mm}$ rostral, $1.6 \mathrm{~mm}$ lateral, and $6.0 \mathrm{~mm}$ ventral to the bregma) (Paxinos and Watson, 1998). In the off-site control experiments, rats were implanted bilaterally with guide cannulas into the ventromedial striatum $(-0.3 \mathrm{~mm}$ rostral, $3.0 \mathrm{~mm}$ lateral, and $5.0 \mathrm{~mm}$ ventral to the bregma). After surgery, the rats were housed individually in cages. The rats were allowed to recover for at least $5 \mathrm{~d}$ and were handled for 1-2 min each day for 3 consecutive days before behavioral experiments. For microinjection, 33-gauge stainless-steel injection cannulas (o.d., $0.2 \mathrm{~mm}$; i.d., 0.08 $\mathrm{mm}$ ) were inserted bilaterally into the guide cannulas; the injection cannulas protruded $1.5 \mathrm{~mm}$ from the tip of guide cannulas to reach the vBNST or ventromedial striatum injection sites. Injection cannulas were attached to a microinfusion pump (CMA/Microdialysis) via PE8 tubing (Natsume Seisakusho). Drugs or vehicle were bilaterally administrated in a volume of $0.5 \mu \mathrm{l}$ per side at a rate of $0.5 \mu \mathrm{l} / \mathrm{min}$, and the injection cannulas were left in place for an additional $1 \mathrm{~min}$ after microinjection to prevent drug backflow.

CPA tests. The CPA test was conducted as described previously (Tanimoto et al., 2003; Deyama et al., 2007a,b). We used a shuttle box composed of two equal-sized compartments $(30 \times 30 \times 30 \mathrm{~cm})$ with distinct tactile and visual cues (one compartment was black with a smooth floor and the other was white with a textured floor) under dim illumination ( $25 \pm 5$ lux at the center of the box). On day 1 (habituation session) and day 2 (preconditioning session), the rats freely explored the two compartments for $900 \mathrm{~s}$; the time spent in each compartment during the exploring period was measured automatically (KN-80; Natsume Seisakusho). Rats that spent $>80 \%$ ( $>720 \mathrm{~s}$ ) of the total time in one side on day 2, or that showed a difference of $>200 \mathrm{~s}$ in the time spent in one side between days 1 and 2, were eliminated from the following procedures. In addition, after the behavioral tests, histological analyses were performed, and data from the rats with misplacements of the bilateral microinjection cannulas were eliminated from the statistical analyses. Both before and after such eliminations, no significant difference ( $p>0.05, n=261$, and $p>0.05, n=185$, respectively) was observed between the time spent in the black ( $448 \pm 7.5$ and $444 \pm 7.2 \mathrm{~s}$, respectively) and white $(451 \pm 7.5$ and $454 \pm 7.2 \mathrm{~s}$, respectively) compartments, indicating no significant bias in compartment preference before conditioning. 
In the CPA test for pain-induced aversion, we used a bias-like protocol (Tzschentke, 1998). Specifically, we designated the compartment in which each rat spent more time ( $>450 \mathrm{~s}$ ) on day 2 (preconditioning session) as each animal's pain-paired compartment. This type of protocol was successfully used to examine the CPA induced by opioid withdrawal (Kosten, 1994; Rafieian-Kopaei et al., 1995; Nakagawa et al., 2005), and by visceral and somatic pain (Tanimoto et al., 2003; Deyama et al., 2007a,b). On day 3, conditioning was performed as follows: in the vehicle control session (conducted between 9:00 A.M. and 12:00 P.M.), each rat was given an injection of saline $(100 \mu l)$ into the left hind paw, and was immediately confined in the non-pain-paired compartment for $1 \mathrm{~h}$. After at least $4 \mathrm{~h}$, each rat was injected with timolol $(1 \mathrm{or} 10 \mathrm{nmol} /$ side), betaxolol ( 1 or $3 \mathrm{nmol} / \mathrm{side}$ ), ICI118,551 (1 or 3 nmol/side), RpcAMPS ( 4 or $40 \mathrm{nmol} / \mathrm{side}$ ), or vehicle into the bilateral vBNST. Ten minutes after the intra-vBNST injection, in the pain-conditioning session (conducted between 2:00 P.M. and 6:00 P.M.), the rats were given an intraplantar injection of $2 \%$ formalin $(100 \mu \mathrm{l})$ into the right hind paw, and then confined in the pain-paired compartment for $1 \mathrm{~h}$. On day 4 , in the test session, each rat was allowed to explore the two compartments freely, and the time spent in each compartment during the exploring period (900 s) was recorded. The CPA scores were calculated by subtracting the time spent in the pain-paired compartment during the test session from the time spent in this compartment during the preconditioning session.

In the experiments investigating intra-vBNST isoproterenol-induced aversion, we designated the compartment in which the rat spent more time ( $>450 \mathrm{~s}$ ) on day 2 (preconditioning session) as the drug-paired compartment for each animal. On days $3-5$, conditioning was performed during 3 consecutive days as follows: the rats were divided into two groups (groups 1 and 2). In the morning session (conducted between 9:00 A.M. and 12:00 P.M.), the rats in group 1 were given an intra-vBNST injection of $\operatorname{drug}(s)$ or vehicle and were immediately confined in the drug-paired compartment for $30 \mathrm{~min}$. However, the rats in group 2 were confined in the non-drug-paired compartment for $30 \mathrm{~min}$ without being given an intra-vBNST injection. In the afternoon session (conducted between 2:00 P.M. and 6:00 P.M.), the rats in group 1 were confined in the non-drug-paired compartment for $30 \mathrm{~min}$ without being given an intra-vBNST injection, and the rats in group 2 were given an intravBNST injection of drug $(\mathrm{s})$ or vehicle and were immediately confined in the drug-paired compartment for $30 \mathrm{~min}$. Each animal received the same conditioning for 3 consecutive days. On day 6 , in the test session, each rat was allowed to explore the two compartments freely, and the time spent in each compartment during the exploring period (900 s) was recorded. The CPA scores were calculated as in the CPA test for pain-induced aversion.

Formalin test. Intraplantar-formalin-induced nociceptive behaviors were measured in a blind manner, as described previously (Tanimoto et al., 2003; Deyama et al., 2007a). Briefly, each rat was placed in a Plexiglas cylinder ( $30 \mathrm{~cm}$ diameter; $30 \mathrm{~cm}$ high) for $30 \mathrm{~min}$, to acclimatize it to the experimental environment. Drug or vehicle was bilaterally injected into the vBNST of each rat, and the animals were returned to the cylinder. Ten minutes after the intra-vBNST injection, the rats were given an intraplantar injection of $2 \%$ formalin $(100 \mu \mathrm{l})$ into the right hind paw and immediately returned to the cylinder. The amount of time the rat spent elevating, licking, shaking, or biting the injected paw was measured for each 5 min period during $60 \mathrm{~min}$. Nociception was quantified using the rating scale method by assigning weights to the following categories of nociceptive behaviors: category 0 , weight is evenly distributed among all paws; category 1, injected paw is lifted; category 2, injected paw is licked, shaken, and bitten. The nociceptive score was calculated for each $5 \mathrm{~min}$ $(300 \mathrm{~s})$ period using the following formula: nociceptive score $=\{$ [time (in seconds) spent with lifting the injected paw] $\times 1+$ [time (in seconds) spent with licking, shaking or biting the injected paw] $\times 2\} / 300$ (in seconds).

Histology. After in vivo microdialysis experiments and behavioral tests, histological analyses were performed. Briefly, the rats were decapitated and the brain was rapidly removed and frozen in powdered dry ice. Coronal sections $(50 \mu \mathrm{m})$ were prepared on a cryostat, thaw-mounted onto slides, stained with thionin, and examined under a microscope $(\times 40)$.

Statistical analyses. The data are expressed as means \pm SEM. CPA scores were compared using a one-way ANOVA followed by the Newman-Keuls post hoc test, when comparing more than two groups, or the Student's $t$ test, when comparing two groups. Two-way ANOVA followed by Bonferroni's post hoc test was used for the statistical analysis of the data from the measurements of nociceptive behaviors. In vivo microdialysis data were assessed using a two-way ANOVA followed by Bonferroni's post hoc test, whereas data for the AUC of extracellular noradrenaline levels were compared by using Student's $t$ test. Differences with $p<$ 0.05 were considered significant.

\section{Results}

\section{Histology}

Our present findings were compatible with previous immunohistochemical studies (Woulfe et al., 1990; Fendt et al., 2005), which revealed the localization of $\mathrm{DBH}$-immunoreactive nerve terminals dominantly in the vBNST (Fig. $1 A$ ).

After the in vivo microdialysis experiments and behavioral tests, histological analyses were performed. Data from rats with correct placements of the microdialysis probe (Fig. $1 B, D$ ) or the bilateral microinjection cannulas (Fig. $1 C, E-I$ ) were used for the statistical analyses.

\section{Pain-induced noradrenaline release within the vBNST}

The baseline concentration of noradrenaline was $0.74 \pm 0.06$ $\mathrm{pg} / 15 \mu \mathrm{l}$, which was compatible with previous microdialysis studies in the BNST (Pacak et al., 1995) and the central amygdala (Watanabe et al., 2003). Direct depolarizing stimulation to neuronal cells by perfusing a high- $\mathrm{K}^{+}$solution increased noradrenaline release repetitively (Fig. $2 A$ ). This high- $\mathrm{K}^{+}$-evoked noradrenaline release was abolished by perfusing a $\mathrm{Ca}^{2+}$-free Ringer's solution. Intraplantar injection of formalin produced an increase in extracellular noradrenaline levels within the vBNST (Fig. 2B). Two-way ANOVA demonstrated a significant effect between the formalin- and saline-injected rats $\left(F_{(1,9)}=8.51 ; p<\right.$ $0.05)$ and a significant interaction between treatment and time $\left(F_{(10,90)}=2.84 ; p<0.01\right)$. A significant increase in the noradrenaline level was observed at $45-60 \mathrm{~min}$ and $60-75 \mathrm{~min}(137 \pm$ $10.1 \%$ and $130 \pm 7.9 \%$; ${ }^{* *} p<0.001$ and ${ }^{* *} p<0.01$, respectively, compared with the saline-injected control group, Bonferroni's post hoc test) after the formalin injection. AUC data also revealed a significant increase ( $p<0.05$, Student's $t$ test) in the noradrenaline level for the formalin-injected group (129 \pm $7.1 \%)$ compared with the saline-injected control group (100 \pm $6.2 \%)$.

\section{Effect of intra-vBNST injection of nonselective $\boldsymbol{\beta}$ - adrenoceptor antagonist on F-CPA}

To determine the role of intra-vBNST $\beta$-adrenergic transmission in the affective component of pain, we examined the effects of timolol ( 1 and $10 \mathrm{nmol} /$ side) injected into the bilateral vBNST on F-CPA. In the intra-vBNST vehicle-injected rats, the time spent in the pain-paired compartment during the test session was $391 \pm 47.5 \mathrm{~s}$, which was significantly shorter $(p<0.05$, Student's $t$ test) than the time during the preconditioning session $(533 \pm$ $14.0 \mathrm{~s}$ ) (Fig. $3 A$ ). In the intra-vBNST timolol ( 1 and $10 \mathrm{nmol} /$ side)-injected rats, no significant differences ( $p>0.05$, Student's $t$ test) were observed in the time spent in the pain-paired compartment between the test $(479 \pm 30.6 \mathrm{~s}$ and $526 \pm 35.7 \mathrm{~s}$, respectively) and preconditioning $(526 \pm 21.9 \mathrm{~s}$ and $515 \pm 19.5 \mathrm{~s}$, respectively) sessions. CPA scores showed the dose-dependent inhibition of F-CPA by intra-vBNST injection of timolol (Fig. 


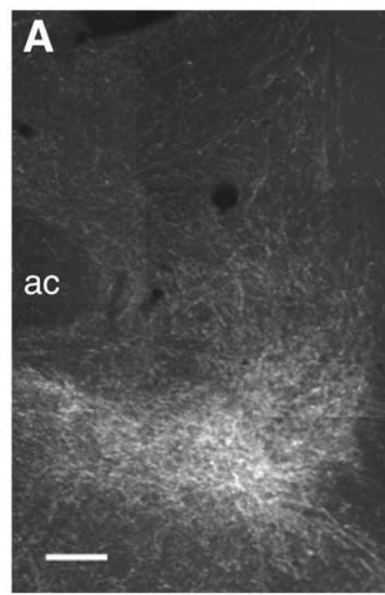

B

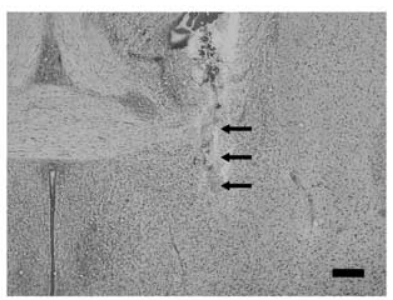

C
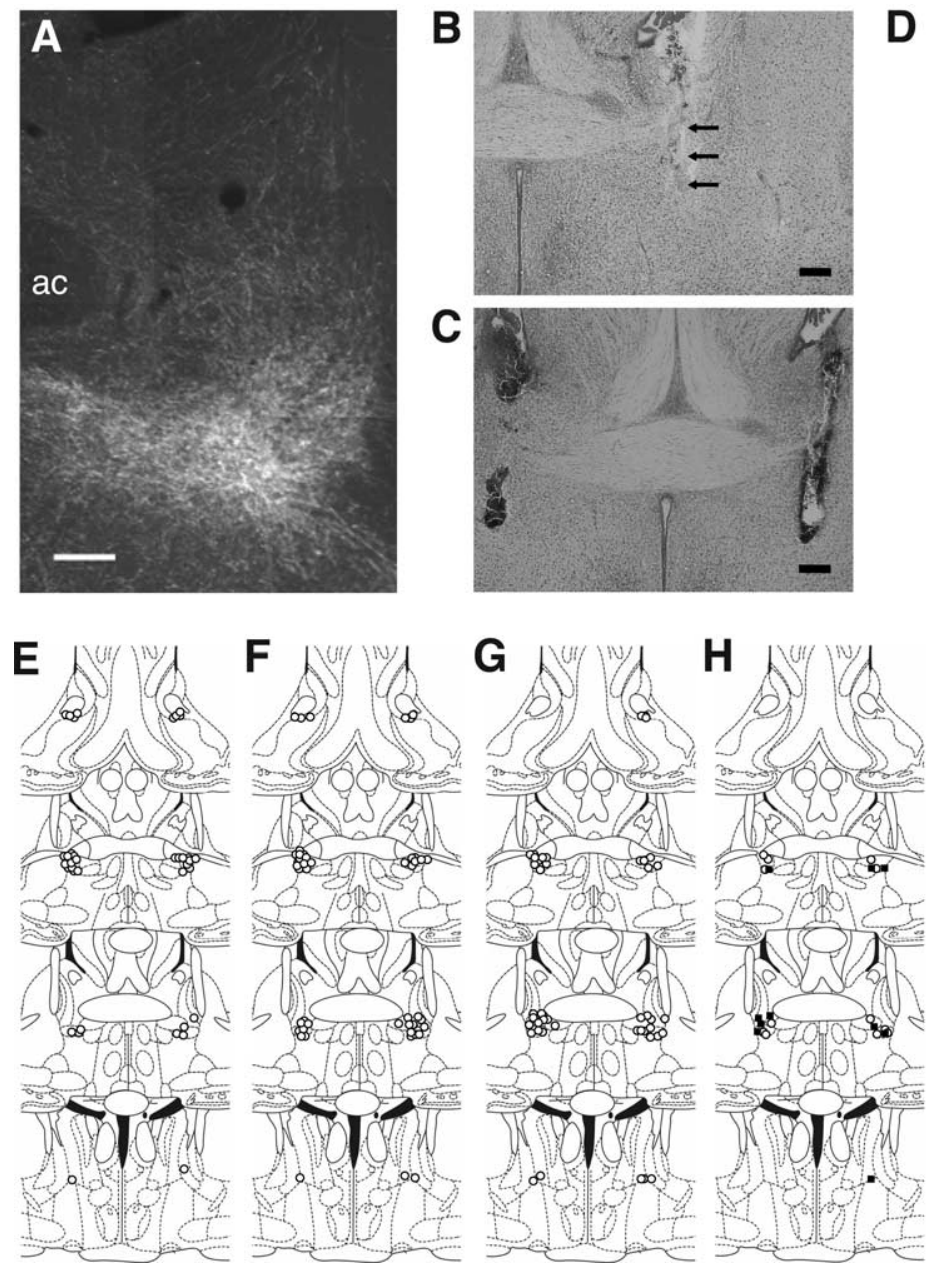

Figure 1. A, Microphotograph of a coronal section of the rat brain showing the DBH-immunoreactivity dominantly located in the ventral part of the BNST. ac, Anterior commissure. Scale bar, $200 \mu \mathrm{m} . \boldsymbol{B}, \boldsymbol{C}$, Representative microphotographs of the microdialysis (arrows; $\boldsymbol{B}$ ) and microinjection sites (C). Scale bars, $300 \mu \mathrm{m}$. D-I, Illustrations demonstrating the placement of the tips of microdialysis probes $(\boldsymbol{D})$ and microinjection cannulas $(\boldsymbol{E}-\boldsymbol{I})(10 \mathrm{nmol}$ timolol, open circles, $\boldsymbol{E} ; 3 \mathrm{nmol}$ betaxolol, open circles, $\boldsymbol{F} ; 3$ nmol ICl118,551, open circles, G; $30 \mathrm{nmol}$ isoproterenol, open circles; $30 \mathrm{nmol}$ isoproterenol plus $40 \mathrm{nmol}$ Rp-cAMPS, filled squares, $\boldsymbol{H} ; 40 \mathrm{nmol}$ Rp-cAMPS, open circles, I). The illustrations of coronal sections were taken from the atlas of Paxinos and Watson (1998); $0.2,-0.26,-0.4$, and -0.8 indicate distances (in millimeters) from bregma.

3B). One-way ANOVA indicated a significant difference among groups $\left(F_{(2,14)}=4.65 ; p<0.05\right)$. Post hoc comparison by the Newman-Keuls test showed that timolol at a dose of $10 \mathrm{nmol} /$ side $(-10.8 \pm 22.2 \mathrm{~s} ; p<0.05)$, but not $1 \mathrm{nmol} /$ side $(47.0 \pm$ $40.8 s ; p>0.05)$, significantly attenuated F-CPA compared with the vehicle-injected group $(143 \pm 36.8 \mathrm{~s})$.

To examine whether intra-vBNST injection of timolol or vehicle per se produced conditioned place preference (CPP) or $\mathrm{CPA}$, timolol (10 nmol/side) or vehicle was injected into the bilateral vBNST of the rats in the absence of intraplantar formalin injection. In both the intra-vBNST vehicle $(n=8)$ - and timolol $(n=6)$-injected groups, no significant differences $(p>0.05$, Student's $t$ test) were observed in the time spent in the painpaired compartment between the test $(513 \pm 12.4 \mathrm{~s}$ and $494 \pm$ $45.6 \mathrm{~s}$, respectively) and preconditioning ( $504 \pm 24.5 \mathrm{~s}$ and $524 \pm$ $20.0 \mathrm{~s}$, respectively) sessions. CPA scores were $-7.63 \pm 28.6 \mathrm{~s}$ and $30.2 \pm 52.7 \mathrm{~s}$, respectively. These data showed that neither CPP nor CPA was induced by intra-vBNST injection of vehicle or timolol, indicating that timolol alone has no motivational effect when injected into the vBNST at this dose.

Intraplantar injection of formalin induced biphasic nocicep-
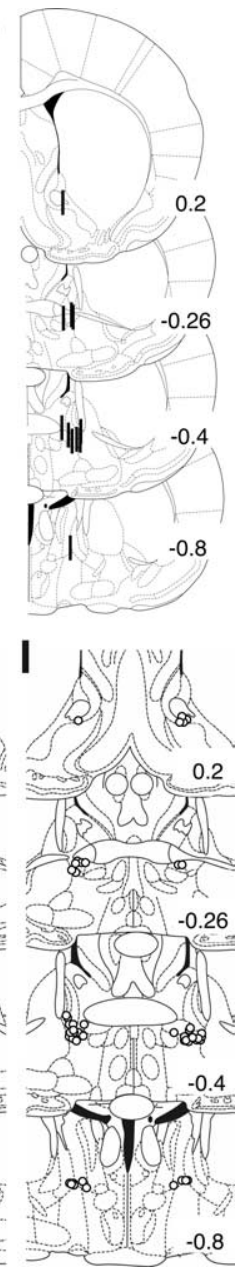

tive responses such as lifting, licking, shaking, and biting. Intra-vBNST injection of timolol at 1 and $10 \mathrm{nmol} /$ side did not affect the formalin-induced nociceptive behaviors compared with the vehicle-injected group (Fig. 4). Two-way ANOVA revealed no significant treatment effect $\left(F_{(2,17)}=\right.$ $0.15 ; p>0.05)$ and no significant interaction between treatment and time $\left(F_{(22,187)}\right.$ $=0.57 ; p>0.05)$.

Effects of intra-vBNST injection of $\boldsymbol{\beta}_{1}$ and $\beta_{2}$-selective antagonists on F-CPA To determine the receptor subtype, the effects of intra-vBNST injection of betaxolol $\left(\beta_{1}\right.$-selective antagonist) or ICI118,551 ( $\beta_{2}$-selective antagonist) on F-CPA were examined. In the intra-vBNST vehicleinjected rats, the time spent in the painpaired compartment during the test session was $396 \pm 23.1 \mathrm{~s}$, which was significantly shorter $(p<0.001$, Student's $t$ test) than the time during the preconditioning session $(524 \pm 14.7 \mathrm{~s})$ (Fig. $5 A$ ). In the intra-vBNST betaxolol ( 1 and $3 \mathrm{nmol} /$ side)-injected rats, no significant differences ( $p>0.05$, Student's $t$ test) were observed in the time spent in the pain-paired compartment between the test (455 \pm $24.5 \mathrm{~s}$ and $485 \pm 40.2 \mathrm{~s}$, respectively) and preconditioning $(515 \pm 18.5 \mathrm{~s}$ and $537 \pm$ $23.3 \mathrm{~s}$, respectively) sessions. Also in the intra-vBNST ICI118,551 ( 1 and $3 \mathrm{nmol} /$ side)-injected rats, no significant differences ( $p>0.05$, Student's $t$ test) were observed in the time spent in the pain-paired compartment between the test (474 \pm $48.2 \mathrm{~s}$ and $563 \pm 38.0 \mathrm{~s}$, respectively) and preconditioning $(532 \pm 29.7 \mathrm{~s}$ and $554 \pm$ 22.8 s, respectively) sessions. CPA scores showed the attenuation of F-CPA by intravBNST injection of these drugs (Fig. $5 B$ ). One-way ANOVA indicated a significant difference among groups $\left(F_{(4,37)}=3.76 ; p<0.05\right)$. A post hoc comparison by the Newman-Keuls test showed that ICI118,551 at a dose of $3 \mathrm{nmol} /$ side $(-9.3 \pm 29.9 \mathrm{~s} ; p<0.01)$, but not ICI118,551 at a dose of 1 $\mathrm{nmol} /$ side $(58.2 \pm 33.9 \mathrm{~s} ; p>0.05)$ or betaxolol at doses of 1 and $3 \mathrm{nmol} /$ side $(60.0 \pm 18.2$ and $52.0 \pm 28.2 \mathrm{~s}$, respectively; $p>$ $0.05)$, significantly attenuated F-CPA compared with the vehicleinjected group $(127 \pm 19.9 \mathrm{~s})$.

To examine whether intra-vBNST injection of betaxolol or ICI118,551 per se produced CPP or CPA, these drugs $(3 \mathrm{nmol} /$ side) were injected into the bilateral vBNST of the rats in the absence of intraplantar formalin injection. In both the intravBNST betaxolol $(n=8)$ - and ICI118,551 $(n=10)$-injected groups, no significant differences $(p>0.05$, Student's $t$ test $)$ were observed in the time spent in the pain-paired compartment between the test $(544 \pm 48.6 \mathrm{~s}$ and $578 \pm 24.4 \mathrm{~s}$, respectively) and preconditioning $(537 \pm 31.0 \mathrm{~s}$ and $571 \pm 17.8 \mathrm{~s}$, respectively) sessions. CPA scores were $-7.63 \pm 35.6 \mathrm{~s}$ and $-6.40 \pm 31.2 \mathrm{~s}$, respectively. These data showed that neither CPP nor CPA was induced by intra-vBNST injection of these drugs, indicating that 


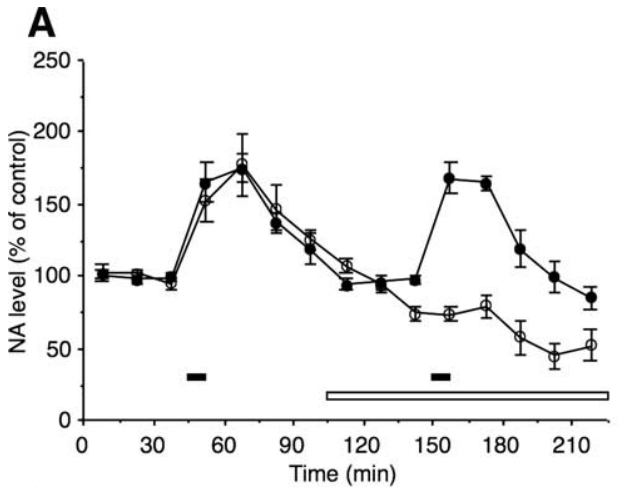

\section{B}

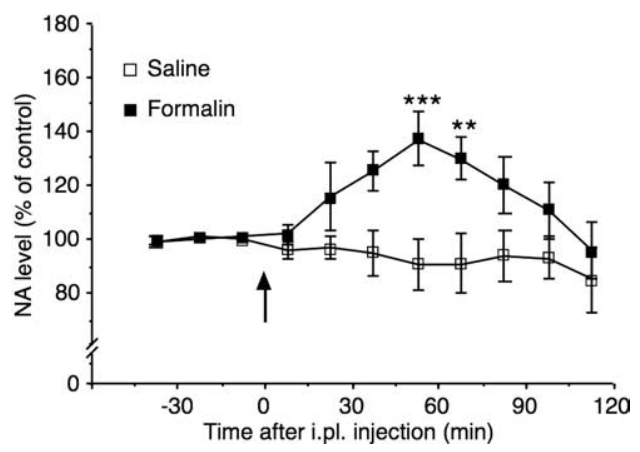

Figure 2. High $\mathrm{K}^{+}$- and pain-induced noradrenaline release in the vBNST. $\boldsymbol{A}, \mathrm{High}-\mathrm{K}^{+}$evoked noradrenaline release in the vBNST of freely moving rats in the presence (closed circle, $n=3$ ) or absence (open circle, $n=3$ ) of $\mathrm{Ca}^{2+}$. Two 7.5 min pulses of a high concentration of $\mathrm{K}^{+}(50 \mathrm{~mm})$ are indicated by closed bars. Perfusion with $\mathrm{Ca}^{2+}$-free Ringer's solution $\left(\mathrm{CaCl}_{2}\right.$ was replaced by an equimolar concentration of $\left.\mathrm{MgCl}_{2}\right)$ is indicated by an open bar. $\boldsymbol{B}$, Effects of intraplantar injection of formalin $(n=5)$ or saline $(n=6)$ on the extracellular noradrenaline levels in the vBNST were examined. The arrow indicates the time point of intraplantar injection. Data are expressed as mean $\pm S E M$ of the percentage basal value, which was calculated as an average of three consecutive dialysates before intraplantar injection or high- $\mathrm{K}^{+}$stimulation. ${ }^{* *} p<0.01,{ }^{* * *} p<0.001$ compared with the saline-injected control group (Bonferroni's post hoc test).

these drugs have no motivational effect when injected into the vBNST at this dose.

As shown in Figure 6, intra-vBNST injection of betaxolol ( 1 or $3 \mathrm{nmol}$ ) or ICI118,551 (1 or $3 \mathrm{nmol}$ ) did not affect formalininduced nociceptive behaviors compared with the vehicleinjected group. Two-way ANOVA of the data for these groups revealed no significant effect of these drugs $\left(F_{(4,31)}=1.07 ; p>\right.$ $0.05)$ and no significant interaction between the drugs and time $\left(F_{(44,341)}=0.80 ; p>0.05\right)$.

\section{Induction of CPA by intra-vBNST injection of \\ $\boldsymbol{\beta}$-adrenoceptor agonist}

The inhibitory effects of intra-vBNST injection of $\beta$-adrenoceptor antagonists on F-CPA motivated us to examine whether CPA is produced through the direct activation of $\beta$-adrenoceptors by intra-vBNST injection of a $\beta$-agonist. In the intra-vBNST vehicle-injected rats, no significant difference ( $p>$ 0.05 , Student's $t$ test) was detected between the test ( $525 \pm 52.0 \mathrm{~s}$ ) and preconditioning $(548 \pm 29.5 \mathrm{~s})$ sessions in the time spent in the drug-paired compartment. As shown in Figure $7 \mathrm{~A}$, conditioning with intra-vBNST injection of isoproterenol, a $\beta$-adrenoceptor agonist, reduced the time spent in the drugpaired compartment. In rats injected with isoproterenol at doses of 3,10, and $30 \mathrm{nmol} /$ side, the time spent in the drug-paired compartment during the test session $(461 \pm 21.9,459 \pm 27.0 \mathrm{~s}$,
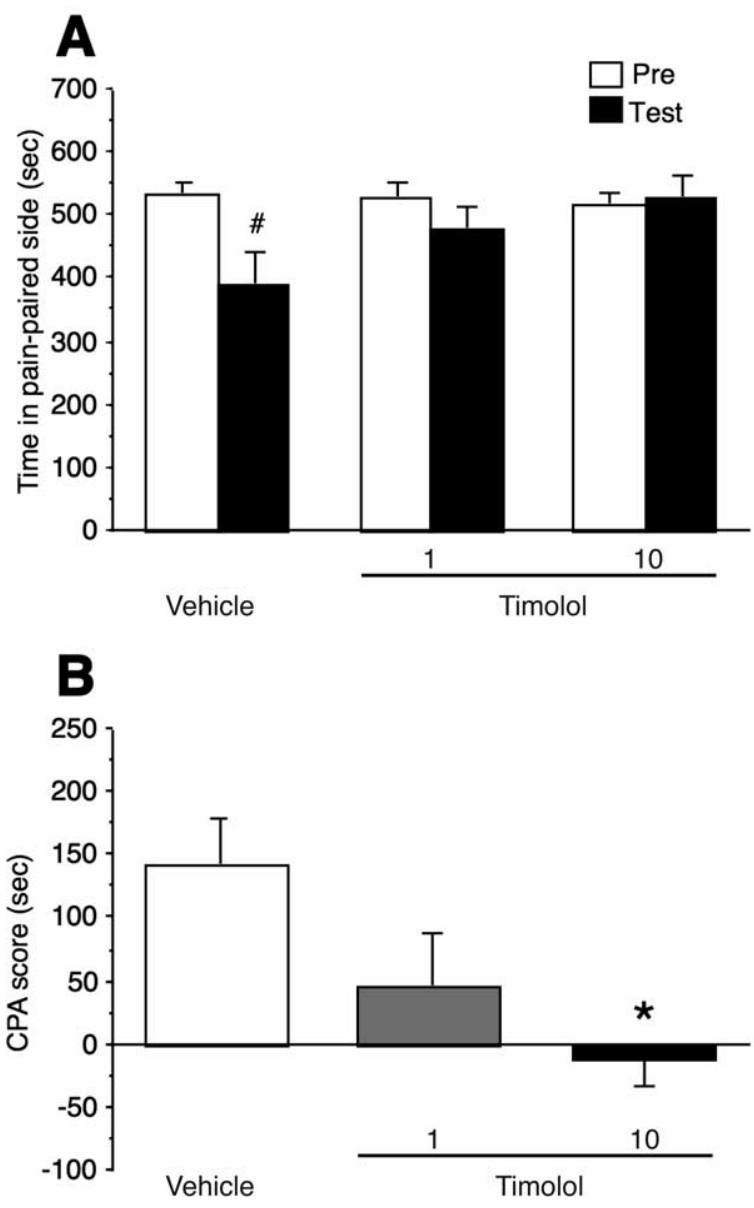

Figure 3. Intra-vBNST injection of timolol dose-dependently attenuated the F-CPA. $\boldsymbol{A}, \boldsymbol{B}$ The columns show the time spent in the pain-paired compartment in the preconditioning (Pre) or test sessions $(\boldsymbol{A})$ and the CPA scores $(\boldsymbol{B})$ of intra-vBNST vehicle- $(n=6)$ or timolol-injected (1 $\mathrm{nmol}, n=6 ; 10 \mathrm{nmol}, n=5$ ) rats. Data are expressed as mean \pm SEM. ${ }^{\#} p<0.05$ compared with the preconditioning session (Student's $t$ test); ${ }^{*} p<0.05$ compared with the vehicleinjected rats (Newman-Keuls post hoc test).

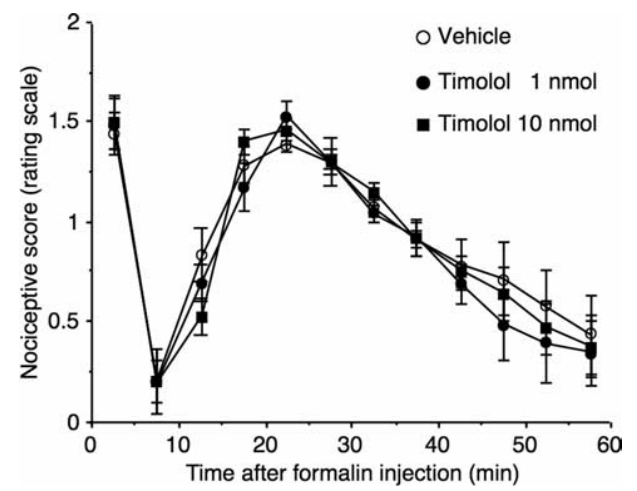

Figure 4. Effects of intra-vBNST timolol on the intraplantar-formalin-induced nociceptive behaviors. Nociceptive scores of the formalin-induced nociceptive behaviors in intra-vBNST vehicle- $(n=6)$ or timolol-injected $(1 \mathrm{nmol}, n=6 ; 10 \mathrm{nmol}, n=8)$ rats. Data are expressed as mean \pm SEM.

and $355 \pm 39.7 \mathrm{~s}$, respectively) was shorter than that during the preconditioning session $(496 \pm 8.96 \mathrm{~s}, 550 \pm 26.7 \mathrm{~s}$, and $550 \pm$ $33.7 \mathrm{~s}$, respectively). A significant reduction in the time spent in the drug-paired compartment was observed in rats treated with 

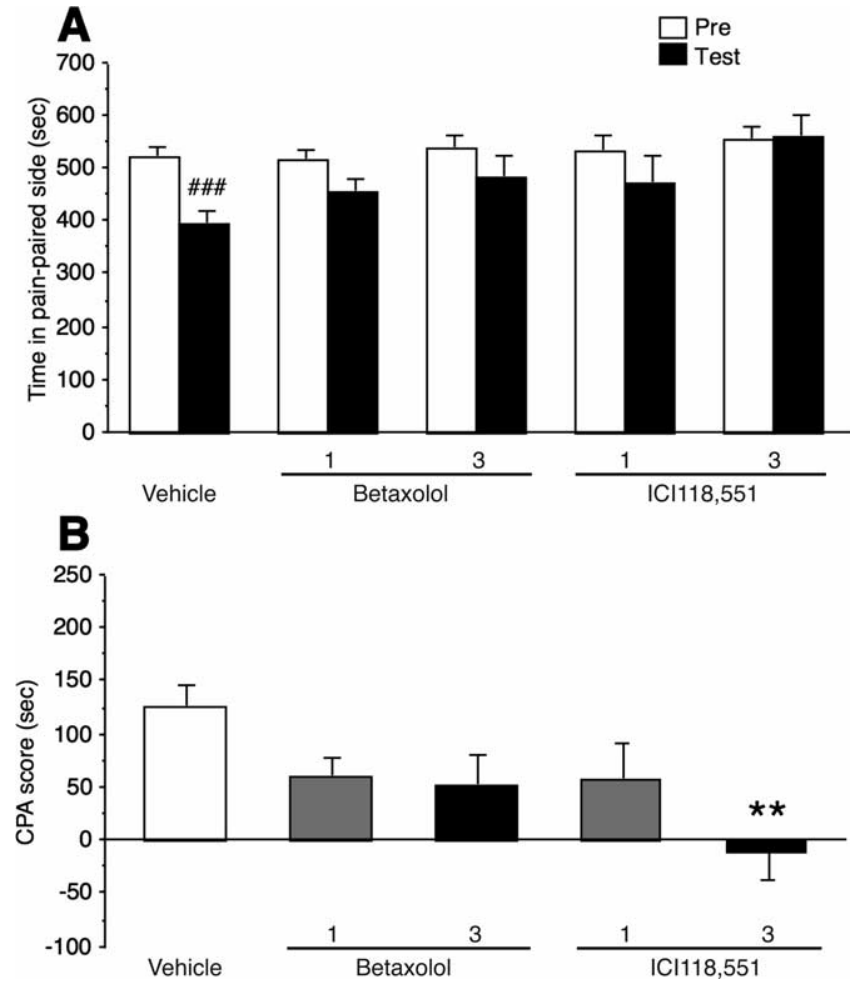

Figure 5. Effects of intra-vBNST injections of betaxolol or ICI118,551 on the F-CPA. $A, B$, The columns show the time spent in the pain-paired compartment in the preconditioning (Pre) or test sessions $(\boldsymbol{A})$ and the CPA scores $(\boldsymbol{B})$ of rats injected with intra-vBNST vehicle $(n=10)$, betaxolol ( $1 \mathrm{nmol}, n=10 ; 3 \mathrm{nmol}, n=9)$, or ICI118,551 ( $1 \mathrm{nmol}, n=6 ; 3 \mathrm{nmol}, n=7)$. Data are expressed as mean \pm SEM. ${ }^{\# \# \# p}<0.001$ compared with the preconditioning session (Student's $t$ test); ${ }^{* *} p<0.01$ compared with the vehicle-injected rats (Newman-Keuls post hoc test).

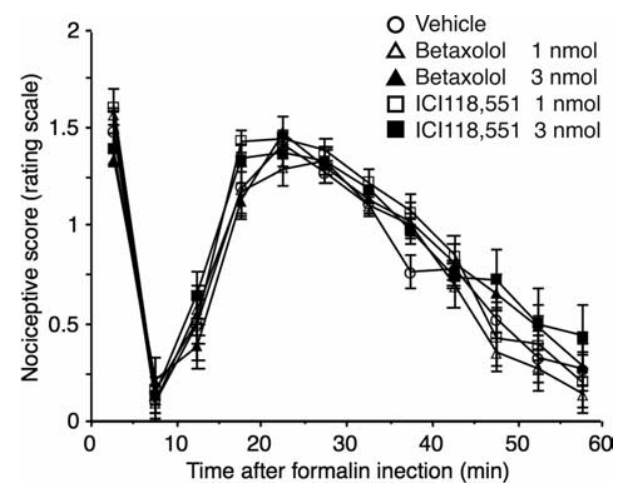

Figure 6. Effects of intra-vBNST betaxolol or ICI118,551 on intraplantar-formalin-induced nociceptive behaviors. Nociceptive scores of the formalin-induced nociceptive behaviors in rats injected with intra-vBNST vehicle $(n=7)$, betaxolol ( $1 \mathrm{nmol}, n=9 ; 3 \mathrm{nmol}, n=7)$, or ICI118,551 ( $1 \mathrm{nmol}, n=7 ; 3 \mathrm{nmol}, n=6)$. Data are expressed as mean \pm SEM.

10 and $30 \mathrm{nmol} /$ side isoproterenol $(p<0.05$ and $p<0.01$, respectively, Student's $t$ test).

CPA scores revealed the dose-dependent induction of CPA by intra-vBNST injection of isoproterenol (Fig. $7 B$ ). One-way ANOVA indicated a significant difference among groups $\left(F_{(4,24)}\right.$ $=3.97 ; p<0.05)$. Post hoc comparison by the Newman-Keuls test showed that isoproterenol at a dose of $30 \mathrm{nmol} / \mathrm{side}(195 \pm$ $59.9 \mathrm{~s} ; p<0.05$ ), but not $3 \mathrm{nmol} /$ side (35.4 $\pm 16.1 \mathrm{~s} ; p>0.05$ ) or $10 \mathrm{nmol} / \mathrm{side}(94.8 \pm 29.1 \mathrm{~s} ; p>0.05)$, significantly induced CPA compared with the vehicle-injected group (22.2 $\pm 27.7 \mathrm{~s})$.
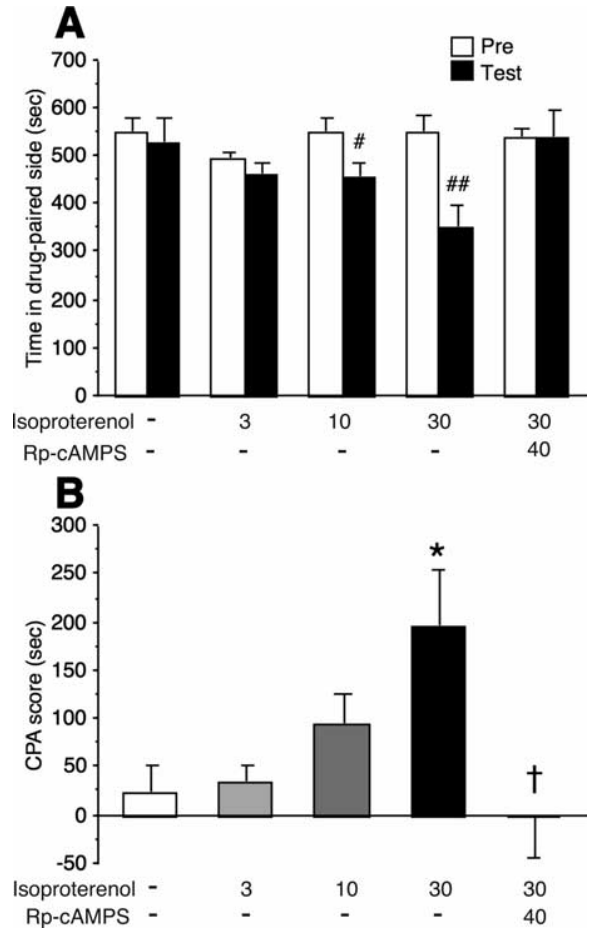

Figure 7. Effects of intra-vBNST injections of vehicle $(n=5)$, isoproterenol ( $3 \mathrm{nmol}, n=5$; $10 \mathrm{nmol}, n=8 ; 30 \mathrm{nmol}, n=6$ ), or $30 \mathrm{nmol}$ of isoproterenol plus $40 \mathrm{nmol}$ of Rp-CAMPS ( $n=$ 5 ) were examined using the place conditioning paradigm. $\boldsymbol{A}, \boldsymbol{B}$, The columns show the time spent in the drug-paired compartment during the preconditioning (Pre) or test sessions $(\boldsymbol{A})$ and the CPA scores (B). Data are expressed as mean \pm SEM. ${ }^{\#} p<0.05$, ${ }^{\# \#} p<0.01$ compared with the preconditioning session (Student's $t$ test); ${ }^{*} p<0.05$ compared with the vehicle-injected group; ${ }^{\dagger} p<0.05$ compared with the isoproterenol ( $30 \mathrm{nmol}$ )-injected group (Newman-Keuls post hoc test).

\section{Effect of PKA inhibitor on isoproterenol-induced CPA}

We examined the effect of coadministering a PKA inhibitor RpcAMPS on intra-vBNST isoproterenol-induced CPA. IntravBNST isoproterenol (30 nmol/side) did not induce CPA when coadministered with Rp-cAMPS (40 nmol/side). No significant difference ( $p>0.05$, Student's $t$ test) was seen in the time spent in the drug-paired compartment between the test $(540 \pm 55.2 \mathrm{~s})$ and preconditioning $(539 \pm 16.6 \mathrm{~s})$ sessions (Fig. $7 A)$. The CPA score $(-1.60 \pm 43.1 \mathrm{~s})$ in the group coadministered with isoproterenol and Rp-cAMPS was significantly $(p<0.05$, NewmanKeuls post hoc test) reduced compared with the CPA score in the group injected with isoproterenol alone (195 $\pm 59.9 \mathrm{~s})$ (Fig. 7B).

To examine whether intra-vBNST repetitive injections of RpcAMPS per se produced CPP or CPA, this drug $(40 \mathrm{nmol} / \mathrm{side}$; $n=6$ ) was injected into the bilateral vBNST of the rats in the absence of isoproterenol. No significant differences $(p>0.05$, Student's $t$ test) were observed in the time spent in the drugpaired compartment between the test ( $535 \pm 28.2 \mathrm{~s})$ and preconditioning $(516 \pm 15.1 \mathrm{~s})$ sessions. CPA score was $-18.2 \pm 20.6 \mathrm{~s}$. These data showed that neither CPP nor CPA was induced by intra-vBNST repetitive injections of Rp-cAMPS in the absence of isoproterenol, indicating that Rp-cAMPS per se has no motivational effect when injected into the vBNST at this dose.

\section{Effect of intra-vBNST injection of PKA inhibitor on F-CPA}

Intra-vBNST vehicle-injected rats spent $409 \pm 25.5 \mathrm{~s}$ in the painpaired compartment during the test session, which was significantly shorter ( $p<0.01$, Student's $t$ test) than the time spent in the pain-paired compartment during the preconditioning ses- 

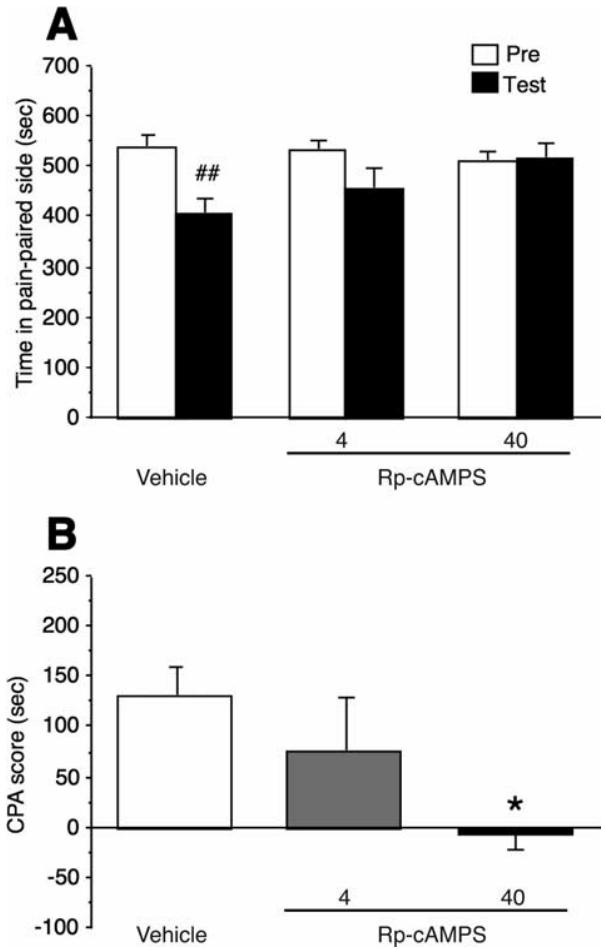

Figure 8. Intra-vBNST injection of Rp-cAMPS dose-dependently attenuated the F-CPA. $A, B$, The columns show the time spent in the pain-paired compartment during the preconditioning (Pre) or test sessions $(\boldsymbol{A})$ and the (PA scores $(\boldsymbol{B})$ of intra-VBNST vehicle- $(n=6)$ or Rp-cAMPSinjected ( $4 \mathrm{nmol}, n=7 ; 40 \mathrm{nmol}, n=7$ ) rats. Data are expressed as mean \pm SEM. ${ }^{\# \#} p<0.01$ compared with the preconditioning session (Student's $t$ test); ${ }^{*} p<0.05$ compared with the vehicle-injected rats (Newman-Keuls post hoc test).

sion $(541 \pm 21.5 \mathrm{~s}$ ) (Fig. 8 A). In intra-vBNST Rp-cAMPS ( 4 and $40 \mathrm{nmol} /$ side)-injected rats, we found no significant differences ( $p>0.05$, Student's $t$ test) in the time spent in the pain-paired compartment between the test $(454 \pm 40.2 \mathrm{~s}$ and $517 \pm 25.0 \mathrm{~s}$, respectively) and preconditioning $(532 \pm 16.3 \mathrm{~s}$ and $511 \pm 17.2 \mathrm{~s}$, respectively) sessions. CPA scores indicated a dose-dependent inhibition of F-CPA by intra-vBNST injection of Rp-cAMPS (Fig. $8 B$ ). One-way ANOVA demonstrated a significant difference among groups $\left(F_{(2,17)}=3.70, p<0.05\right)$. Post hoc comparison with the Newman-Keuls test showed that Rp-cAMPS at a dose of $40 \mathrm{nmol} / \mathrm{side}(-5.71 \pm 17.5 \mathrm{~s} ; p<0.05)$, but not 4 $\mathrm{nmol} /$ side $(77.6 \pm 51.6 \mathrm{~s} ; p>0.05)$, significantly attenuated F-CPA compared with the vehicle-injected group (132 $\pm 26.4 \mathrm{~s}$ ).

To examine whether intra-vBNST single injection of RpcAMPS per se produced CPP or CPA, this drug (40 nmol/side; $n=7$ ) was injected into the bilateral vBNST of the rats in the absence of intraplantar formalin injection. No significant differences ( $p>0.05$, Student's $t$ test) were observed in the time spent in the pain-paired compartment between the test $(511 \pm 49.2 \mathrm{~s})$ and preconditioning $(504 \pm 18.5 \mathrm{~s})$ sessions. CPA score was $-7.14 \pm 58.4 \mathrm{~s}$. These data showed that neither CPP nor CPA was induced by intra-vBNST injection of Rp-cAMPS in the absence of intraplantar formalin injection, indicating that Rp-cAMPS per se has no motivational effect when injected into the vBNST at this dose.

As shown in Figure 9, intra-vBNST injection of Rp-cAMPS (4 and $40 \mathrm{nmol}$ ) did not affect formalin-induced nociceptive behaviors compared with the vehicle-injected group. Two-way ANOVA of the data for these groups revealed no significant effect of intra-vBNST Rp-cAMPS injection $\left(F_{(2,14)}=0.38 ; p>0.05\right)$

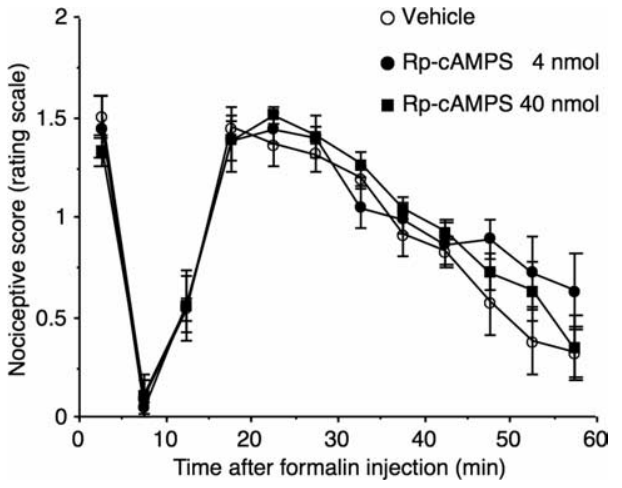

Figure 9. Effects of intra-vBNST Rp-cAMPS on the intraplantar-formalin-induced nociceptive behaviors. Nociceptive scores of the formalin-induced nociceptive behaviors in intra-vBNST vehicle- $(n=6)$ or Rp-cAMPS-injected $(4 \mathrm{nmol} ; n=5,40 \mathrm{nmol} ; n=6)$ rats. Data are expressed as means \pm SEM.

and no significant interaction between intra-vBNST injection and time $\left(F_{(22,154)}=1.08 ; p>0.05\right)$.

\section{Effects of intrastriatum injection of $\beta$-antagonists and PKA inhibitor on F-CPA}

Off-site control injections of $\beta$-antagonists or PKA inhibitor into the ventromedial striatum had no significant effect on F-CPA (Fig. 10). In all groups, intraplantar injection of formalin significantly shortened the time spent in the pain-paired compartment during the test session (vehicle, $406 \pm 23.4 \mathrm{~s}, n=7$; timolol, $379 \pm 48.3 \mathrm{~s}, n=6$; betaxolol, $355 \pm 28.9 \mathrm{~s}, n=5$; ICI118,551, $435 \pm 30.0 \mathrm{~s}, n=7$; Rp-cAMPS, $392 \pm 20.7 \mathrm{~s}, n=7$ ) compared with the time during the preconditioning session (vehicle, $546 \pm$ $26.2 \mathrm{~s}$; timolol, $494 \pm 15.7 \mathrm{~s}$; betaxolol, $511 \pm 35.1 \mathrm{~s}$; ICI118,551, $558 \pm 21.8 \mathrm{~s}$; Rp-cAMPS, $502 \pm 13.5$ s) (Fig. 10 B). One-way ANOVA on the data for the CPA scores of these groups (vehicle, $140 \pm 23.1 \mathrm{~s}$; timolol, $114 \pm 52.0 \mathrm{~s}$; betaxolol, $156 \pm 22.6 \mathrm{~s}$; ICI118,551, $123 \pm 30.4 \mathrm{~s}$; Rp-cAMPS, $110 \pm 26.3 \mathrm{~s})$ revealed no significant effect of intrastriatum injections of these drugs $\left(F_{(4,27)}\right.$ $=0.228 ; p>0.05)$ (Fig. 10C).

\section{Discussion}

We demonstrated previously that excitotoxic lesions of the BNST abolished F-CPA without reducing nociceptive behaviors (Deyama et al., 2007a). Our current study using a conditioned place paradigm and an in vivo microdialysis technique extends those findings by showing that enhanced noradrenergic transmission via $\beta$-adrenoceptors within the vBNST plays a key role in the negative affective component of pain. In addition, intravBNST injection of a PKA inhibitor suppressed F-CPA, showing the involvement of PKA in pain-induced aversion. To our knowledge, this study is the first to provide evidence of the crucial role of PKA activation within the BNST in the development of negative emotion.

Because the CPA test used in this study was based on associative learning between a noxious stimulus-induced aversive affect and a neutral environmental context, whether the attenuation of CPA was caused by the impairment of associative learning or the suppression of the primary aversive affect is difficult to determine. In the present study, we demonstrated that intra-vBNST injection of isoproterenol, a $\beta$-adrenoceptor agonist, produced CPA even in the absence of formalin-induced noxious stimulus. This indicates that the activation of $\beta$-adrenoceptors within the vBNST is sufficient to produce the negative affective states. 

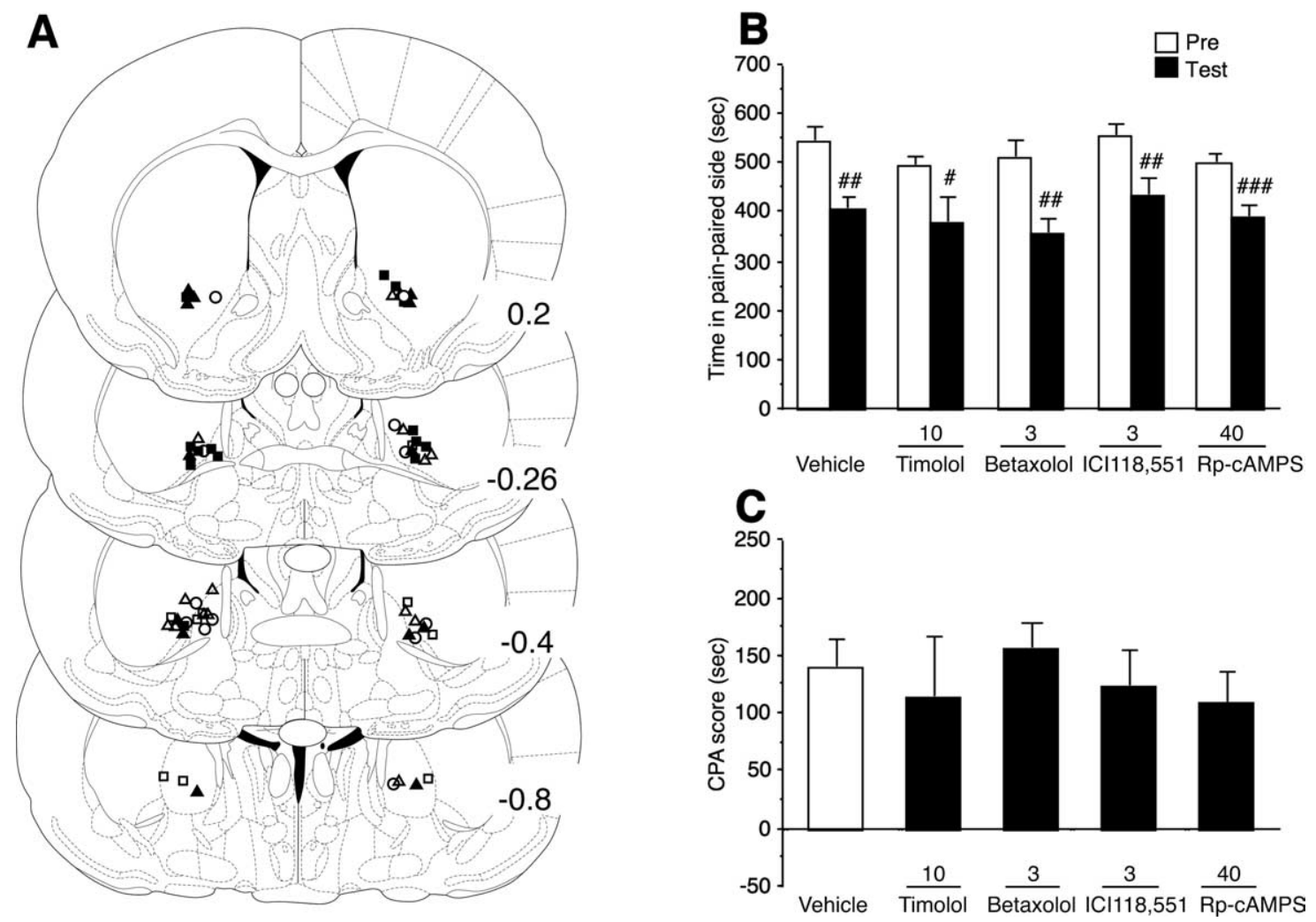

Figure 10. Off-site control injections of $\beta$-antagonists or PKA inhibitor into the ventromedial striatum did not affect the F-CPA. $A$, Illustrations demonstrating the placement of the tips of microinjection cannulas (vehicle, filled triangles; $10 \mathrm{nmol}$ timolol, open circles; $3 \mathrm{nmol}$ betaxolol, open squares; $3 \mathrm{nmol}$ ICl118,551, filled squares; $40 \mathrm{nmol}$ Rp-cAMPS, open triangles). $\boldsymbol{B}, \boldsymbol{C}$, The columns show the time spent in the pain-paired compartment in the preconditioning or test sessions $(\boldsymbol{B})$ and the CPA scores $(\boldsymbol{C})$ of rats injected with vehicle $(n=7)$, timolol $(n=6)$, betaxolol $(n=$ 5), ICl 118,551 ( $n=7)$, or Rp-cAMPS ( $n=7)$. Data are expressed as mean \pm SEM. ${ }^{\#} p<0.05,{ }^{\# \#} p<0.01,{ }^{\# \#} p<0.001$ compared with the preconditioning session (Student's $t$ test).

Therefore, the attenuation of F-CPA by the blockade of $\beta$-adrenoceptors within the vBNST may be caused by the reduction of the primary aversive affect.

Important roles of intra-BNST noradrenergic transmission in negative emotional states such as anxiety, fear, and aversive responses have been reported (Delfs et al., 2000; Cecchi et al., 2002; Walker et al., 2003; Fendt et al., 2005). Specifically, Fendt et al. (2005) reported that exposure to trimethylthiazoline, a component of fox odor, increased noradrenaline release in rats, and that intra-vBNST administration of the $\alpha_{2}$-adrenoceptor agonist clonidine suppressed both the enhanced release of noradrenaline and trimethylthiazoline-induced potentiation of freezing behavior. Cecchi et al. (2002) showed that noradrenaline release within the BNST of rats was increased by acute restraint stress, and that intra-vBNST injection of a mixture of $\beta_{1}$ - and $\beta_{2}$-adrenoceptor antagonists blocked stress-induced anxiety-like behaviors in the elevated plus-maze test. Furthermore, Delfs et al. (2000) reported that microinjection of $\beta$-antagonists or a $\alpha_{2}$-agonist into the BNST markedly attenuated opiate withdrawal-induced CPA in rats. They also demonstrated that lesions of the ventral noradrenergic bundle suppressed opiate withdrawal-induced CPA, suggesting the involvement of noradrenergic fibers arising from the A1/A2 medullary cell group. In this study, we demonstrated the enhanced release of noradrenaline within the vBNST during formalin-induced noxious stimulus and the involvement of $\beta$-adrenoceptors in pain-induced aversion. These findings strongly suggest a pivotal role of intra-vBNST noradrenergic transmission via $\beta$-adrenoceptors in the development of negative affective states.

In previous studies, a mixture of betaxolol and ICI118,551 was used to examine the roles of intra-vBNST $\beta$-adrenoceptors in the opiate withdrawal-induced CPA (Delfs et al., 2000) and stressinduced anxiety-like behaviors (Cecchi et al., 2002), and, thus, these did not distinguish between $\beta_{1}$ - and $\beta_{2}$-adrenoceptors. We examined the effects of these antagonists separately and found that intra-vBNST injection of ICI118,551 dose-dependently attenuated F-CPA. This compound completely suppressed F-CPA at a dose of $3 \mathrm{nmol} /$ side. However, betaxolol attenuated F-CPA, but it was only a partial effect even at a dose of $3 \mathrm{nmol} / \mathrm{side}$. These data suggest the critical role of intra-vBNST $\beta_{2}$-adrenoceptors in the negative affective component of pain, although the partial contribution of $\beta_{1}$-adrenoceptors cannot be excluded.

Pain itself is a major stress that induces negative emotion, HPA-axis activation, and an increase in blood pressure. In this study, we focused on the emotional aspect of pain, and demonstrated the crucial role of intra-BNST noradrenergic transmission in pain-induced aversion. As for HPA-axis activation, Cecchi et al. (2002) reported that intra-BNST injection of an $\alpha_{1^{-}}$ adrenoceptor antagonist, but not $\beta$-adrenergic antagonists, attenuated immobilization stress-induced adrenocorticotropic hormone secretion. Regarding cardiovascular responses, it has been reported that electrical stimulation of the medial BNST increases mean arterial pressure (Dunn and Williams, 1995), and microinjection of corticotropin-releasing factor (CRF), a peptide implicated in negative emotional states, into this region enhances heart rate (Nijsen et al., 2001). These findings suggest that intraBNST noradrenergic and/or CRFergic transmission may be involved in pain-induced HPA-axis activation and cardiovascular responses. This possibility presents an issue to investigate in future studies. 
$\beta$-Adrenoceptors couple to adenylate cyclases to activate PKA. Thus, we examined the role of PKA activation within the vBNST in isoproterenol- and formalin-induced CPA. The intravBNST isoproterenol-induced CPA was reversed by the coadministration of a PKA inhibitor, Rp-cAMPS. Furthermore, intravBNST injection of Rp-cAMPS dose-dependently attenuated the F-CPA. However, intra-vBNST injection of Rp-cAMPS did not reduce formalin-induced nociceptive behaviors. These results indicate that the PKA activation within the vBNST via the enhanced $\beta$-adrenergic transmission mediates the negative affective, but not sensory, component of pain.

Injections of $\beta$-antagonists or PKA inhibitor into the ventromedial striatum, the brain region close to the BNST, had no effect on F-CPA. These results, together with the observation of intense innervations of noradrenergic fibers into the vBNST, suggest that the vBNST is the likely site of action of drugs examined in this study. However, additional experiments are needed to clarify the target neurons for noradrenergic fibers and the substrates for PKA within the vBNST. Nevertheless, we have uncovered some of the neuronal mechanisms underlying the affective component of pain by showing the involvement of an enhanced $\beta$-adrenoceptor-PKA signaling pathway within the vBNST in pain-induced aversion. Because chronic pain is frequently associated with psychological and emotional dysfunction (McWilliams et al., 2003), studies of the neural circuits and the molecular mechanisms involved in the affective component of pain may have considerable clinical importance in the treatment of chronic pain.

\section{References}

Cecchi M, Khoshbouei H, Javors M, Morilak DA (2002) Modulatory effects of norepinephrine in the lateral bed nucleus of the stria terminalis on behavioral and neuroendocrine responses to acute stress. Neuroscience 112:13-21.

Delfs JM, Zhu Y, Druhan JP, Aston-Jones G (2000) Noradrenaline in the ventral forebrain is critical for opiate withdrawal-induced aversion. Nature 403:430-434.

Deyama S, Nakagawa T, Kaneko S, Uehara T, Minami M (2007a) Involvement of the bed nucleus of the stria terminalis in the negative affective component of visceral and somatic pain in rats. Behav Brain Res 176:367-371.

Deyama S, Yamamoto J, Machida T, Tanimoto S, Nakagawa T, Kaneko S, Satoh M, Minami M (2007b) Inhibition of glutamatergic transmission by morphine in the basolateral amygdaloid nucleus reduces pain-induced aversion. Neurosci Res 59:199-204.

Dunn JD, Williams TJ (1995) Cardiovascular responses to electrical stimulation of the bed nucleus of the stria terminalis. J Comp Neurol 352:227-234.

Fendt M, Siegl S, Steiniger-Brach B (2005) Noradrenaline transmission within the ventral bed nucleus of the stria terminalis is critical for fear behavior induced by trimethylthiazoline, a component of fox odor. J Neurosci 25:5998-6004.

Forray MI, Gysling K, Andrés ME, Bustos G, Araneda S (2000) Medullary noradrenergic neurons projecting to the bed nucleus of the stria terminalis express mRNA for the NMDA-NR1 receptor. Brain Res Bull 52:163-169.

Johansen JP, Fields HL (2004) Glutamatergic activation of anterior cingulated cortex produces an aversive teaching signal. Nat Neurosci 7:398-403.

Johansen JP, Fields HL, Manning BH (2001) The affective component of pain in rodents: direct evidence for a contribution of the anterior cingulate cortex. Proc Natl Acad Sci U S A 98:8077-8082.

Kosten TA (1994) Clonidine attenuates conditioned aversion produced by naloxone-precipitated opiate withdrawal. Eur J Pharmacol 254:59-63.

Lei LG, Sun S, Gao YJ, Zhao ZQ, Zhang YQ (2004) NMDA receptors in the anterior cingulated cortex mediate pain-related aversion. Exp Neurol 189:413-421.

McWilliams LA, Cox BJ, Enns MW (2003) Mood and anxiety disorders associated with chronic pain: an examination in a nationally representative sample. Pain 106:127-133.

Nakagawa T, Yamamoto R, Fujio M, Suzuki Y, Minami M, Satoh M, Kaneko S (2005) Involvement of the bed nucleus of the stria terminalis activated by the central nucleus of the amygdala in the negative affective component of morphine withdrawal in rats. Neuroscience 134:9-19.

Nijsen MJ, Croiset G, Diamant M, De Wied D, Wiegant VM (2001) CRH signaling in the bed nucleus of the stria terminalis is involved in stressinduced cardiac vagal activation in conscious rats. Neuropsychopharmacology 24:1-10.

Pacak K, McCarty R, Palkovits M, Kopin IJ, Goldstein DS (1995) Effects of immobilization on in vivo release of norepinephrine in the bed nucleus of the stria terminalis in conscious rats. Brain Res 688:242-246.

Paxinos G, Watson C (1998) The rat brain in stereotaxic coordinates. San Diego: Academic.

Rafieian-Kopaei M, Gray AM, Spencer PS, Sewell RD (1995) Contrasting actions of acute or chronic paroxetine and fluvoxamine on morphine withdrawal-induced place conditioning. Eur J Pharmacol 275:185-189.

Tanimoto S, Nakagawa T, Yamauchi Y, Minami M, Satoh M (2003) Differential contributions of the basolateral and central nuclei of the amygdala in the negative affective component of chemical somatic and visceral pains in rats. Eur J Neurosci 18:2343-2350.

Tzschentke TM (1998) Measuring reward with the conditioned place preference paradigm: a comprehensive review of drug effects, recent progress and new issues. Prog Neurobiol 56:613-672.

Walker DL, Toufexis DJ, Davis M (2003) Role of the bed nucleus of the stria terminalis versus the amygdala in fear, stress, and anxiety. Eur J Pharmacol 463:199-216.

Watanabe T, Nakagawa T, Yamamoto R, Maeda A, Minami M, Satoh M (2003) Involvement of noradrenergic system within the central nucleus of the amygdala in naloxone-precipitated morphine withdrawal-induced conditioned place aversion in rats. Psychopharmacology (Berl) 170:80-88.

Woulfe JM, Flumerfelt BA, Hrycyshyn AW (1990) Efferent connections of the A1 noradrenergic cell group: a DBH immunohistochemical and PHA-L anterograde tracing study. Exp Neurol 109:308-322. 